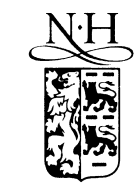

\title{
Characterization of optical UV filters using Rutherford backscattering spectroscopy
}

\author{
R. Vlastou ${ }^{\text {a,* }}$, E. Fokitis ${ }^{\text {a }}$, S. Maltezos ${ }^{\text {a }}$, G. Kalliabakos ${ }^{\text {b }}$, M. Kokkoris ${ }^{\text {, }}$, \\ E. Kossionides ${ }^{b}$ \\ a Department of Physics, National Technical University of Athens, Zografou Campus, GR-157 80 Athens, Greece \\ ${ }^{\mathrm{b}}$ Institute of Nuclear Physics, NRCPS Demokritos, GR-153 10, Greece
}

\begin{abstract}
Rutherford backscattering spectroscopy (RBS) has been used during the development of multilayer thin film optical filters, especially designed for the fluorescence detector of the Pierre AUGER Project. Depth profiles of the heavy components have been measured directly, while reliable results for the light components could also be extracted indirectly. Relative thickness and density of the individual layers and deviations from the desired thickness and stoichiometry have been deduced. The findings of the RBS method have been compared and discussed with results obtained by other characterization techniques. (C) 2000 Elsevier Science B.V. All rights reserved.
\end{abstract}

PACS: $68.55 . \mathrm{Jk}$

Keywords: Rutherford backscattering; Thin films; Optical filters

\section{Introduction}

The design and construction of optical UV-filters for the fluorescence detector of the Auger project [1] is of primary importance. The aim of the filters is to increase the signal to noise ratio in detection of nitrogen fluorescence induced in the atmosphere by the highest energy cosmic rays. These cosmic rays interact with the atmospheric air and produce extensive air showers which, in

\footnotetext{
${ }^{*}$ Corresponding author. Tel.: +30 01-772-3008; fax: +30 01772-3025.

E-mail address: vlastou@central.ntua.gr (R. Vlastou).
}

turn, ionize and excite nitrogen atoms. The fluorescence emitted by the nitrogen atoms, molecules and ions covers mainly the range $300-410 \mathrm{~nm}$. This radiation will be subsequently collected and detected by arrays consisted of spherical mirrors, photomultipliers and optical filters placed in front of them, with their passband matching the nitrogen fluorescence region. The functional and technical characteristics of the filters should be: (a) high transmittance in the region $300-410 \mathrm{~nm}$, higher than $85 \%$ in average, which is better than the performance of absorption type filters used in similar experiments. (b) Very low transmittance in the region $410-600 \mathrm{~nm}$, less than $2 \%$ in average. The filters, being able to maximize the signal to 
noise ratio, could thus extend the operation cycle of the fluorescence detector during the night, when the moon light produces high levels of background noise [2]. (c) A minimum possible variation of the optical transmittance with the angle of the direction of the incident light to the photomultiplier collector, which could vary within $15^{\circ}$ (in root mean square value) from the axis perpendicular to the filter surface. (d) Uniform spectral characteristics and mechanical stability in the mass production process.

The required properties of the optical filters led to specific production techniques, such as the dielectric multilayer thin film deposition on UV glass, using high-low refractive index combinations of UV-transparent materials. Several combinations have been tried for the design such as $\mathrm{Al}_{2} \mathrm{O}_{3}, \mathrm{Sc}_{2} \mathrm{O}_{3}, \mathrm{ZrO}_{2}, \mathrm{WO}_{3}$ for high index and $\mathrm{MgF}_{2}, \mathrm{SiO}_{2}$ for low index layers. In order to select the optimal deposition technique for the mass production of these filters, the RBS method has been used to provide information concerning the relative thickness and homogeneity of the individual layers as well as possible deviations from the desired stoichiometry.

\section{Sample production and characterization}

The appropriate software has been developed for the design of the filters [3], to calculate the optical thickness and combination of layers required for an optimal performance. The main deposition technique used to produce 1-12 layer pairs of the mentioned materials, was electron beam deposition, either by reactive evaporation $\left(\sim 10^{-4}\right.$ mbar oxygen) or in very high vacuum $\left(<10^{-5}\right.$ mbar). Several combinations of thin films have been tried with $\mathrm{Al}_{2} \mathrm{O}_{3}, \mathrm{Sc}_{2} \mathrm{O}_{3}, \mathrm{ZrO}_{2}, \mathrm{WO}_{3}$ for high refractive index and $\mathrm{MgF}_{2}, \mathrm{SiO}_{2}$ for low refractive index layers.

During the deposition process, thickness and deposition rates were monitored by a quartz crystal monitor. In addition, after the preparation of the samples, a Tally Step Profilometer was used to measure the total thickness as well as the sum of the thicknesses of the two types of thin films with low and high refractive index, respectively.
The spectral transmittance of the produced filters could be measured afterwards and then fitted with a specific software by considering the optical thickness (product of the geometrical thickness and the refractive index divided by the reference wavelength) of the various layers as free parameters. The extracted average values of the parameters and their standard deviation have been compared with those deduced from the RBS analysis.

XRF measurements have also been performed and provided important information concerning the total concentration of the heavy elements in the layers as well as the impurities of the substrate, which helped the analysis of the RBS spectra.

From all these methods only the total thickness (geometrical or optical) of the layers could be extracted, while with the RBS measurements the relative thickness of the individual layers could be deduced and the total relative thickness could then be compared to the values given by the other techniques and extract information concerning the packing densities of the deposited materials. In addition, the reproducibility of the layer thicknesses, the homogeneity of the films and the reliability of the production methods could be tested with the RBS method.

\section{RBS experiments and results}

For the RBS measurements, a 2.9 or $3.0 \mathrm{MeV}$ alpha beam was used, supplied by the tandem T11/ 25 accelerator at the NRCPS "Demokritos". The detector was Si surface barrier, positioned at $165^{\circ}$ with respect to the beam direction and at a distance of $14 \mathrm{~cm}$ from the target. The data acquisition and control hardware were driven by a personal computer with the use of the appropriate software.

The RBS spectra were analysed by utilizing the computer code RUMP [4]. Typical results for some of the samples are shown in Figs. 1-3 for $\mathrm{WO}_{3} / \mathrm{MgF}_{2}$ (12 pair layers), $\mathrm{ZrO}_{2} / \mathrm{SiO}_{2}$ (8 pair layers) and $\mathrm{ZrO}_{2} / \mathrm{MgF}_{2}$ (6 pair layers), respectively. It is seen that a good fit to the data could be achieved and reliable results could be extracted 


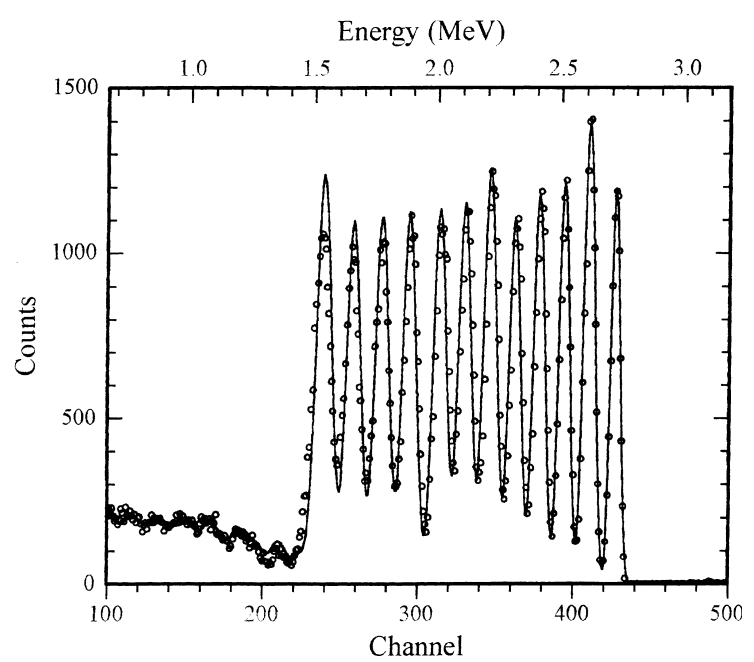

Fig. 1. RBS spectrum of 12 layer pairs of $\mathrm{WO}_{3} / \mathrm{MgF}_{2}$ films on UV glass taken with $3 \mathrm{MeV}{ }^{4} \mathrm{He}$. The 12 strong peaks of $\mathrm{W}$ correspond to the 12 layers of $\mathrm{WO}_{3}$. The solid line represents simulation by the RUMP code.

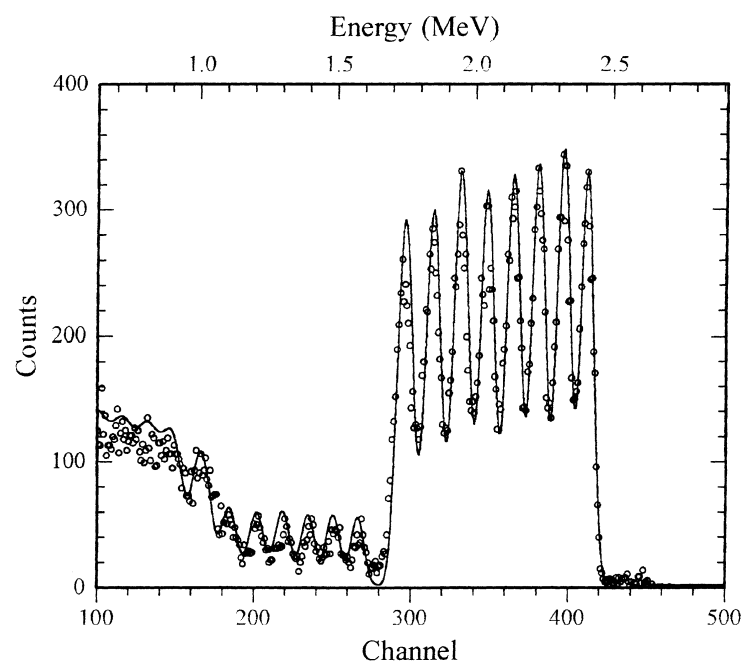

Fig. 2. RBS spectrum of eight layer pairs of $\mathrm{ZrO}_{2} / \mathrm{SiO}_{2}$ films on $\mathrm{UV}$ glass taken with $2.9 \mathrm{MeV}^{4} \mathrm{He}$. The eight strong peaks of $\mathrm{Zr}$ correspond to the eight layers of $\mathrm{ZrO}_{2}$ and the next seven weak peaks of $\mathrm{Si}$ to the seven layers of $\mathrm{SiO}_{2}$. The 8th layer of $\mathrm{SiO}_{2}$ cannot be distinquished from the substrate. The small peaks at about channels 435,450 are due to the heavier isotopes of $\mathrm{Zr}$. The solid line represents simulation by the RUMP code.

(with an uncertainty up to $10 \%$ ) in most samples. The peaks of the heavy elements $(\mathrm{W}, \mathrm{Zr})$ can be clearly separated by the ${ }^{4} \mathrm{He}$ beam and can thus be

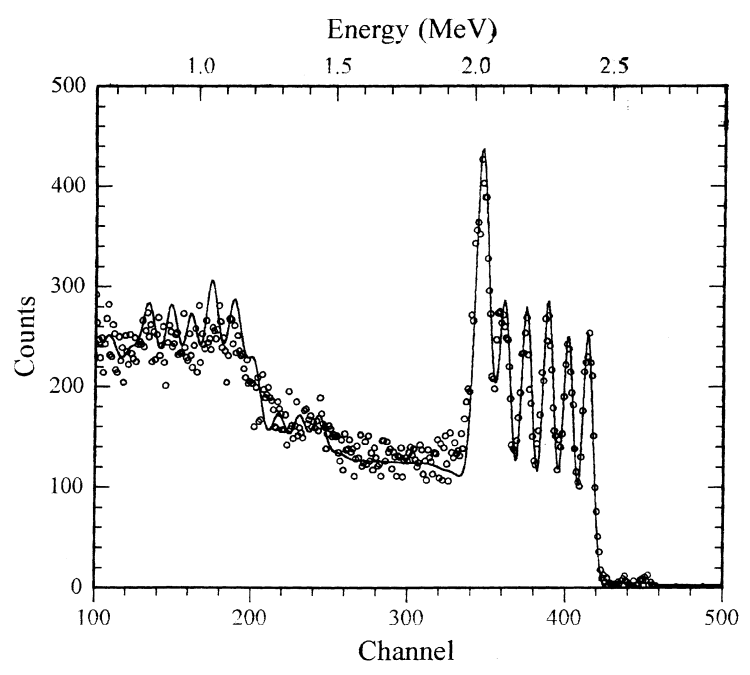

Fig. 3. RBS spectrum of six layer pairs of $\mathrm{ZrO}_{2} / \mathrm{MgF}_{2}$ films on UV glass taken with $2.9 \mathrm{MeV}{ }^{4} \mathrm{He}$. The strong peaks of $\mathrm{Zr}$ correspond to the six layers of $\mathrm{ZrO}_{2}$, the background is due to the $\mathrm{Ba}$ impurities of the substrate and the weak peaks on the $\mathrm{Si}$ substrate correspond to the $\mathrm{Mg}$ and $\mathrm{F}$ of the six $\mathrm{MgF}_{2}$ layers. The small peaks at about channels 435,450 are due to the heavier isotopes of $\mathrm{Zr}$. The solid line represents simulation by the RUMP code.

used to determine the relative thickness of the films (by using the nominal density of the bulk materials) as well as their stoichiometry.

The XRF measurements helped the analysis of the RBS spectra since contributions to the background due to impurities of the substrate, such as $\mathrm{Ca}$ and $\mathrm{Ba}$, could easily be identified. In the samples containing $\mathrm{SiO}_{2}$ layers, as seen in Fig. 2, the layers close to the substrate could not be clearly analysed and the thickness of the first layer on the substrate is given with an uncertainty up to $\sim 100 \%$. In the samples containing $\mathrm{MgF}_{2}$ as low refractive index layers, the $\mathrm{Mg}$ and $\mathrm{F}$ peaks are sitting on the background of the substrate and can only be analysed by using the additional information coming from the valleys between the heavy element peaks. These valleys correspond to the energy loss of the beam passing through the $\mathrm{MgF}_{2}$ layers, so their width and depth depend on the thickness and the stoichiometry of the layers. In the case of $\mathrm{MgF}_{2}$ layers shown in Fig. 3, the thickness of the layers has been established very accurately even for the layer sitting on the sub- 
strate, which doesn't correspond to a valley. The thickness of this layer moves the place of the substrate contribution in the spectrum and especially the edge of the Ba impurity, which provides a sensitive marker for the determination of the properties of the inner layers.

Concerning the stoichiometry of the thin films, the $\mathrm{MgF}_{2}, \mathrm{ZrO}_{2}$ and $\mathrm{SiO}_{2}$ layers were found to follow the desired composition, while in the $\mathrm{WO}_{3}$ layers, oxygen losses of up to $15 \%$ were observed. These results are consistent with the comments presented in [5].

\section{Discussion of the results}

The optimum calculated thicknesses [3] of each of the high and low index materials, were the same (quarterwave) for each material. However, the thickness of the deposited layers were found to vary, due to the instability of the thickness monitor. From the analysis of the RBS spectra, the relative thicknesses of the individual layers exhibit a variation from the average value of the order of $16 \%$ for $\mathrm{ZrO}_{2}, 10 \%$ for $\mathrm{WO}_{3}$ and $\mathrm{SiO}_{2}$ and $9-16 \%$ for $\mathrm{MgF}_{2}$.

During the deposition of dielectric materials a reduced packing density of the films can be produced which may affect the film properties [5]. The packing density $p$ is defined as the ratio of the density of the film material $p_{\mathrm{f}}$ to the bulk material $p_{\mathrm{b}}$. Since the same mass of the material in a film with reduced packing desity, fills a larger volume than a dence film, the geometrical film thickness is enhanced. The hardness, stresses and stability, as well as the optical properties of the films can also be influenced by the packing density. The refractive index $n$ of the film is affected by the lower packing density since the voids of the film have an index $n=1.0$, while by absorbing water vapors when exposed to air, the refractive index of the voids becomes $n=1.33$.

Using the RBS relative thicknesses (by using the density of the bulk material), in conjunction with the thickness measurements of the step profilometer, the following values for the packing density were deduced. For $\mathrm{ZrO}_{2} p=0.66 \pm 0.06$, for $\mathrm{SiO}_{2} p=1.10 \pm 0.15$, for $\mathrm{MgF}_{2} p=0.92 \pm 0.09$ and for $\mathrm{WO}_{3} p=0.92 \pm 0.09$. These results are in good agreement with the values presented in [5], except for $\mathrm{MgF}_{2}$ which exhibits an enhanced packing density, compared with the value of [5].

The actual thicknesses of the layers deduced from RBS and profilometry were introduced in the simulation software to calculate the spectral transmittance of the filters as a function of the wavelength. The calculated values were compared with the experimentally measured values and were found to agree within their uncertainties. The spectral transmittance, however, depends on the refractive index, which is affected by the density of the films. In order to investigate the influence of the relative density variation $\mathrm{d} p_{\mathrm{f}} / p_{\mathrm{f}}$ to the relative variation of the refractive index $\mathrm{d} n / n$, the following expression has been used:

$\frac{\mathrm{d} n}{n}=\frac{\left(n^{2}+2\right)\left(n^{2}-1\right)}{6 n^{2}} \frac{\mathrm{d} p_{\mathrm{f}}}{p_{\mathrm{f}}}$

derived from the Lorentz-Lorenz equation. The deduced values of $\mathrm{d} n / n$ were found to differ from $\mathrm{d} p_{\mathrm{f}} / p_{\mathrm{f}}$ by a factor of 0.4 for the low index materials $(n \approx 1.5)$, while for the high index materials $(n \approx 2.0)$ this factor becomes 0.8 . Thus an uncertainty of the refractive index during the simulation process, of up to $25 \%$ for the low and $12 \%$ for the high index materials, can be considered to be tolerable. An example of the calculated and measured spectral profiles is presented in Fig. 4 for the sample with eight pairs of $\mathrm{ZrO}_{2} / \mathrm{SiO}_{2}$ (shown in Fig. 2). The two curves are estimated to agree within $8 \%$. Besides the experimental errors, the observed differences could also be due to possible density variation within the layers.

\section{Summary}

RBS has been used as a characterization technique during the development of multilayer thin film optical filters especially designed for the fluorescence detector of the Pierre AUGER Project. Several combinations of films with materials of high refractive index, such as $\mathrm{ZrO}_{2}$ and $\mathrm{WO}_{3}$ and low refractive index, such as $\mathrm{MgF}_{2}$ and $\mathrm{SiO}_{2}$ have been examined. Three of them are presented in this 


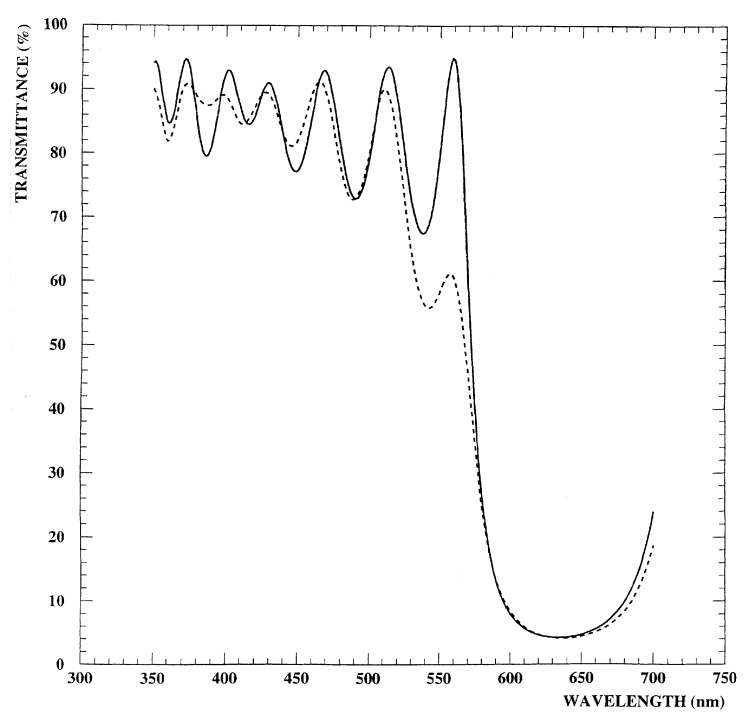

Fig. 4. The spectral transmittance of the sample with eight pairs of $\mathrm{ZrO}_{2} / \mathrm{SiO}_{2}$ (presented in Fig. 2), as a function of the wavelength. The dashed line represents the measured transmittance and the solid line the calculated transmittance using the thicknesses of the layers deduced from RBS and profilometry.

work, namely a sample with 12 layer pairs of $\mathrm{WO}_{3} /$ $\mathrm{MgF}_{2}$ films, another with 8 layer pairs of $\mathrm{ZrO}_{2} /$ $\mathrm{SiO}_{2}$ films and a third with 6 layer pairs of $\mathrm{ZrO}_{2} /$ $\mathrm{MgF}_{2}$ films on UV glass. The relative thickness and the stoichiometry of the individual layers, the thickness variation of the layers, the spectral transmittance of the films, as well as the packing density of materials after the film deposition have been deduced. The extracted values have been compared with the ones taken from the literature or measured with different characterization techniques and have been found to be in good agreement.

\section{Acknowledgements}

It is our pleasure to thank A. Braem of Optics Laboratory, CERN and A. Travlos, of Inst. of Materials Science, NRCPS "Demokritos", for preparing the optical multilayer filters and for useful discussions. Our warmest thanks to A. Karydas of Inst. of Nuclear Physics, NRCPS "Demokritos", who made the XRF measurements and assisted in their interpretations. Acknowledgements are also due to A. Geranios, A. Petrides and M. Vassiliou of the University of Athens, for measuring the spectral transmittance of the filters and K. Patrinos for his contribution in the project.

\section{References}

[1] AUGER Collaboration, Design report of the Pierre AUGER Project, March 1997.

[2] J. Elbert, Proceedings of Tokyo Conference on Techniques for the Study of Extremely High Energy Cosmic Rays (of M. Nagano) 1993, 232.

[3] E. Fokitis, S. Maltezos, E. Papantonopoulos, JHEP. Proceedings of the Euroconference on the Standard Model and beyond, PRHEP-corfou 98/045, September 1998.

[4] L.R. Doolittle, Nucl. Instr. and Meth. B 9 (1985) 344.

[5] E. Ritter, Appl. Opt. 15 (10) (1976) 2318. 